\title{
Global vorticity shedding for a vanishing wing
}

\author{
M. S. Wibawa ${ }^{1}$, S. C. Steele ${ }^{1}$, J. M. Dahl ${ }^{1} \dagger$, D. E. Rival ${ }^{1,2}$, G. D. Weymouth ${ }^{3}$ \\ and M. S. Triantafyllou ${ }^{1}$ \\ ${ }^{1}$ Mechanical Engineering, Massachusetts Institute of Technology, 77 Massachusetts Avenue, \\ Cambridge, MA 02139, USA \\ 2 Mechanical and Manufacturing Engineering, University of Calgary, 2500 University Dr NW, \\ Calgary, AB, T2N 1N4, Canada \\ ${ }^{3}$ Singapore-MIT Alliance for Research and Technology Centre, S16-05-08 3 Science Drive 2, \\ 117543, Singapore
}

(Received 31 March 2011; revised 7 October 2011; accepted 20 December 2011; first published online 13 February 2012)

If a moving body were made to vanish within a fluid, its boundary-layer vorticity would be released into the fluid at all locations simultaneously, a phenomenon we call global vorticity shedding. We approximate this process by studying the related problem of rapid vorticity transfer from the boundary layer of a body undergoing a quick change of cross-sectional and surface area. A surface-piercing foil is first towed through water at constant speed, $U$, and constant angle of attack, then rapidly pulled out of the fluid in the spanwise direction. Viewed within a fixed plane perpendicular to the span, the cross-sectional area of the foil seemingly disappears. The rapid spanwise motion results in the nearly instantaneous shedding of the boundary layer into the surrounding fluid. Particle image velocimetry measurements show that the shed layers quickly transition from free shear layers to form two strong, unequal-strength vortices, formed within non-dimensional time $t^{*}=0.03$, based on the foil chord and forward velocity. These vortices are connected to, and interact with, the foil's tip vortex through additional streamwise vorticity formed during the rapid pulling of the foil. Numerical simulations show that two strong spanwise vortices form from the shed vorticity of the boundary layer. The three-dimensional effects of the foil removal process are restricted to the tip of the foil. This method of vorticity transfer may be used for quickly introducing circulation to a fluid to provide forcing for biologically inspired flow control.

Key words: swimming/flying, vortex flows, wakes/jets

\section{Introduction}

Extreme manoeuvrability in both natural and engineered fluid flow problems can be achieved through the control of vorticity either within the boundary layer or the free fluid. Swifts achieve extreme manoeuvres in flight through rapid changes in wing orientation, which alter the effects of leading-edge vortices acting on the wing (Müller \& Lentink 2004). In such cases where there is a rapid reorientation of a wing in 
the fluid, vorticity from the boundary layer must be transferred to the free fluid as the area of the wing changes. This rapid transfer of vorticity differs from typical vortex shedding, where vorticity is slowly fed into the fluid along a separation line (Taneda 1977).

Klein (1910) first proposed the coffee-spoon thought experiment to show that vortex sheets can be generated in a virtually inviscid fluid when the body disappears; for example, by removing, melting, or dissolving the spoon. Kaden (1931) developed analytic and experimental analogies to the disappearing coffee spoon using a vanishing plate, showing that the rapid production of vortices in fluids of low viscosity is possible through the merging of spiral-shaped vorticity layers emanating from sharp edges. Taylor (1953) provided an analytical treatment for an impulsively accelerated and then vanishing circular disk. Using energy, impulse, and circulation conservation, and assuming that the freed vorticity will form a single vortex ring with uniform core, Taylor determined the resulting vortex ring strength and diameter when the accelerated disk disappears.

Prandtl (1927) and Betz (1950) also analysed the subject of vorticity production in fluids of negligible viscosity, showing that vortices could only be formed by the rapid roll-up of thin vortical layers. Prandtl (1936) used visualization techniques to examine the vortex formation for a variety of unsteady motions and bluff bodies including documenting the transfer of boundary layer vorticity to a free vortex through the rapid removal of a spinning cylinder as its rotation is stopped and the cylinder is quickly accelerated out of frame.

Renewed interest on the topic of boundary layer vorticity being transferred to the surrounding fluid through unsteady motion has emerged in the context of biomimetic propulsion. Buchholz \& Smits (2006), Buchholz \& Smits (2008), von Ellenrieder, Parker \& Soria (2003), and Blondeaux et al. (2005) have studied the wake structures of finite-span flapping foils, obtaining insight into biological propulsors through the simplified problem of pitching and heaving finite-span wings. Childress, Vandenberghe \& Zhang (2006) conducted experiments in an oscillatory flow using a body with flexible surface, resulting in a variable frontal area exposed to the flow. The flapperlike model was found to suspend itself against gravity by taking advantage of a change in added mass between its upstroke and downstroke. Similarly, Spagnolie \& Shelley (2009) ran numerical simulations where the frontal area of a two-dimensional cylinder was varied against the oncoming flow. In this case, propulsive forces were possible by controlling the phase difference between the shape change and the fluid oscillation. In contrast to the passive control in the experiment of Childress et al. (2006), the shape change was actively controlled. In these studies, a change in object area or shape is responsible for the net force on the body, as unequal levels of kinetic energy (and thus added mass force) are imparted by the body in opposing stroke planes. The resulting flow patterns are of great interest as boundary layer vorticity is also shed in the fluid.

In animal locomotion, appendages which are responsible for propulsion may be varied in shape. One example of a fin shape variation is in the duck feet studied by Johansson \& Norberg (2003), where the transition of the duck's foot from a drag orientation to a lift orientation results in the intense shedding of vorticity in the form of a starting vortex. Another elegant example in nature is the propulsion mechanism of water striders at the interface between air and water, as described by Dickinson (2003). In this situation, the leg of the water strider imparts momentum to the fluid under the water surface in order to propel its body above the surface of the water. Similarly, Hsieh \& Lauder (2004) found that basilisk lizards, when running on the water surface, rapidly impart vorticity from their feet into the water, producing dynamic lift and 
supporting the weight of the lizard. The rapid transfer of bound circulation into U-shaped vortices beneath the surface as with the water strider and basilisk lizard was further examined by $\mathrm{Hu} \&$ Bush (2010).

Other studies related to this topic of shape change include the observations of the diving grebe's lobed feet by Johansson \& Norberg (2001), wind-tunnel measurements looking at the variable sweepback angles of swift wings by Lentink et al. (2007) as well as the kinematic studies of Hubel et al. (2009) considering shape variation during the flapping cycle in bat flight. These studies considered shape change from a quasi-steady framework. Most recently Dong et al. (2010) examined the performance of a fish pectoral fin undergoing both active and passive deformations on the paddling stroke. When compared to a rigid fin, a significant improvement in thrust is observed when the deforming fin is allowed to efficiently transfer vorticity to an attached tip vortex.

This paper investigates what happens to the vorticity in the boundary layer of a body when it is released globally, i.e. shed simultaneously from every part of the surface of the body into the fluid. Investigating this process requires the disappearance or dissolution of a body, which is difficult to implement. For this reason we use the related simplified problem of a surface-piercing foil, towed at constant speed and at a constant angle of attack, which is suddenly pulled in the spanwise direction out of the fluid.

\section{Global vorticity shedding}

First we consider the concept of global vorticity shedding, which is the process we associate with a vanishing body in a viscous fluid, when all the boundary layer vorticity is instantaneously released into the flow. This global shedding is to be contrasted with the gradual release of vorticity in flows separating from body surfaces, where vorticity can only be released gradually, as effectively shown by Taneda (1977) and discussed by Morton (1984). Taneda showed that, for example, two-dimensional flow separation from the suction side of an aerofoil, moving at an angle of attack, can only occur at either a single point or at most a few isolated points. His work served to underline the fact that even the 'massive separation' observed in stalled foils is caused by the gradual release of vorticity often from a single separation point; a fact that can be obscured when streamlines are shown rather than streaklines. Taneda's work explains the relatively long time needed before a separation cavity is formed and reiterates the importance of using streaklines to identify the mechanisms of flow separation.

When the aerofoil is rapidly removed from the fluid, as in this study, the boundarylayer vorticity is released in the fluid nearly instantaneously; but this is only an approximation to global vorticity shedding. The process is complicated by the generation of additional streamwise vorticity due to the rapid spanwise motion. We will also refer to this process as global vorticity shedding to distinguish it from the gradual vorticity shedding from a separation line.

The important questions we will address are how vorticity reorganizes, the shape of the newly forming patterns, and the time it takes to reach them. Prandtl's experiment, with a spinning cylinder that is stopped and then accelerated out of view (Prandt 1936), demonstrates the rapid redistribution of vorticity that was initially bound to the spinning cylinder into a single vortex. The question remains of why such a single vortex forms, and whether the same simple patterns arise with more complex body forms. For example, in Taylor's development of a vortex ring out of a vanishing solid 
(a)

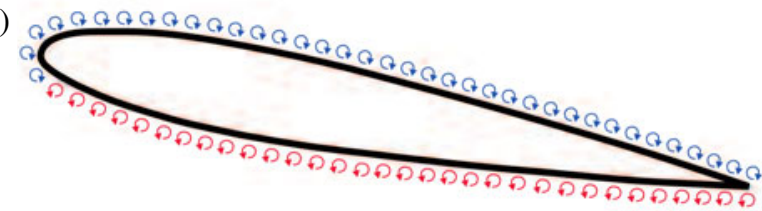

(b)

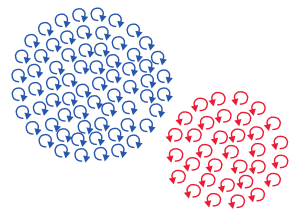

FIGURE 1. (Colour online available at journals.cambridge.org/flm) Vorticity in the boundary layer of the vanishing wing redistributes into two wake vortices, conserving the net circulation: ( $a$ ) boundary-layer vorticity; $(b)$ vortex pair in wake.

disk (Taylor 1953), the theory assumes that there is some unspecified mechanism that will reconfigure the initial vorticity distribution into a single vortex ring. Once this assumption is made, one can obtain the circulation and core size of the ring; however, no proof is provided that the resulting shape should be a ring. However, an experiment that uses a towed rigid disk is difficult to perform, because the flow separates quickly from the sharp edges, forming a long wake; hence the flow does not resemble the potential flow assumed by Taylor.

As a result, we choose to consider the case of a vanishing wing at an angle of attack, which retains the essence of Taylor's problem with a flow that remains largely potential-flow-like. Again, it is not obvious how the boundary-layer vorticity from such a wing will reorganize. We show in this paper that the overall outcome is as sketched in figure 1.

Since the foil vanishes rapidly, the time scales for vorticity reorganization are also small; hence, viscosity does not play a significant role in dissipating vorticity. We can therefore employ arguments of conservation of circulation, which are strictly valid for inviscid flows only. Effects on the flow from the formation of three-dimensional patterns and the possibility of annihilation of opposite-sign vorticity are discussed.

\section{Experiment description and methodology}

In order to study the rapid transfer and redistribution of boundary-layer vorticity from a vanishing body to the free fluid, we employ a vanishing foil that is towed at an angle of attack. First, a finite-span foil is towed at an angle of attack to reach a steady speed, develop bound circulation and generate lift. Next, the foil is pulled rapidly out of the flow in the spanwise direction, and made to 'vanish', leaving the boundary-layer vorticity to redistribute in the free fluid.

The experiment was carried out in a tank with dimensions $2.4 \mathrm{~m} \times 0.75 \mathrm{~m} \times 0.75 \mathrm{~m}$, illustrated in figure 2. A rectangular-tipped NACA 0012 aluminum wing with a chord of $c=0.069 \mathrm{~m}$ and span $b=0.43 \mathrm{~m}$, mounted on a traverse system, was towed along the length of the tank at a constant speed of $U=0.2 \mathrm{~m} \mathrm{~s}^{-1}$ resulting in a Reynolds number based on the chord of $R e=14000$. One end of the wing pierced the free surface of the water, while the wing tip was located near the middle of the tank. The wing was towed at four different angles of attack: $0,5,10$ and $15^{\circ}$. When the wing reached a predefined location within the tank, it was rapidly pulled upwards by a certain distance, equal to 1.44 chord lengths. This motion was actuated using a Copley Controls STA2504 linear motor with $\pm 0.35 \mathrm{~mm}$ accuracy. The wing angle of attack was adjusted with $\pm 0.5^{\circ}$ accuracy using a Newport 481 -A precision rotation stage.

Time-resolved particle image velocimetry (PIV) was used to obtain qualitative and quantitative information about the flow. For PIV measurements, the laser plane was 


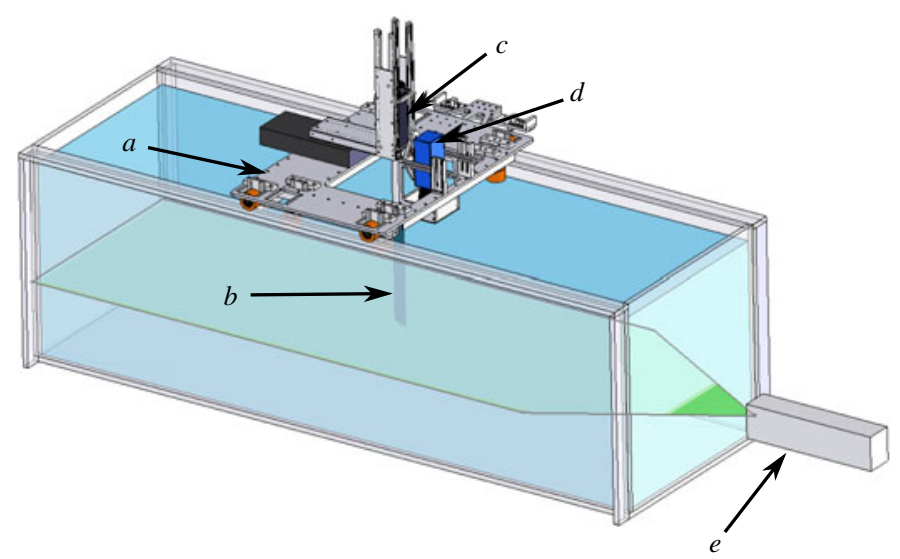

FIGURE 2. (Colour online) Schematic of towing-tank setup with (a) traverse, (b) NACA0012 wing, $(c)$ linear motor, $(d)$ high-speed camera for PIV and $(e)$ laser head for PIV.

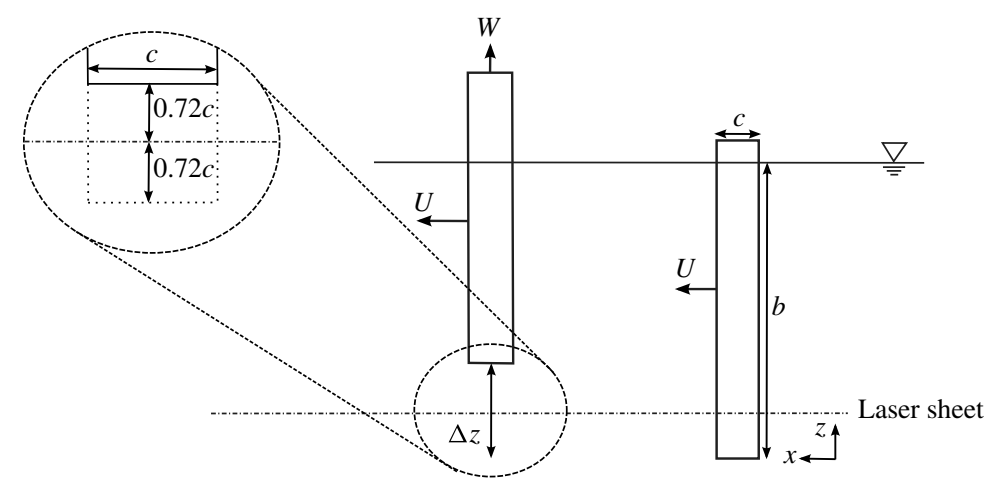

FIGURE 3. Experimental setup: the foil is initially submerged (right) and then rapidly pulled upwards by 1.44 chord lengths (left). Velocity arrows show the direction of foil motion.

placed at 0.72 chord lengths from the tip, at the midpoint of the foil's spanwise excursion.

\subsection{Foil motion}

The wing is initially towed for $\Delta t=4 \mathrm{~s}$ (approximately a distance of $0.8 \mathrm{~m}$ ) from its start position. This distance corresponds to over ten chord lengths of travel and therefore a quasi-steady flow develops (Wagner 1925) before the wing is pulled upwards and vanishes from the PIV measurement plane. The towing at constant speed continues after the wing vanishes from the measurement plane for another $0.7 \mathrm{~m}$, or another ten chord lengths. As shown in figure 3, a wing translation of $\Delta z / c=1.44$ (laser plane located half-way at $\Delta z / c=0.72$ ) was chosen, so that the PIV measurement plane would lie outside of the established tip-vortex core region; see Birch \& Lee (2005). 


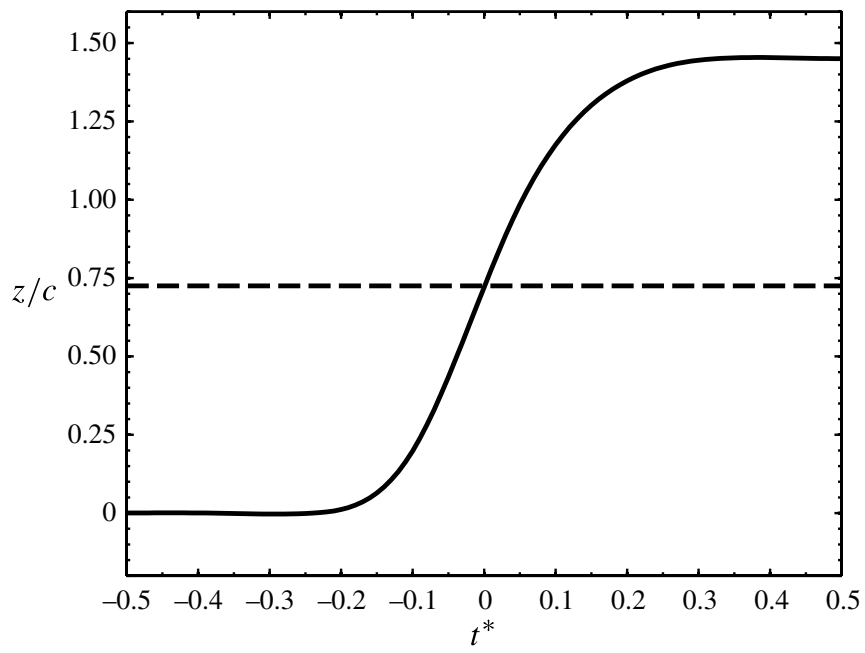

FIGURE 4. Vertical motion profile of the wing tip as a function of the non-dimensional time. Solid line shows the vertical motion profile of the wing tip. Dashed line shows the position of the laser plane with respect to this motion.

The vectorial superposition of the forward motion $(U)$ with the rapid upward motion $(W)$ is described by the following equation:

$$
\boldsymbol{M}\left(t^{*}\right)= \begin{cases}U \boldsymbol{i} & \text { for } t^{*}<-0.25 \\ U \boldsymbol{i}+W \boldsymbol{k} & \text { for }-0.25 \leqslant t^{*}<0.25 \\ U \boldsymbol{k} & \text { for } 0.25 \leqslant t^{*},\end{cases}
$$

where $t^{*}=t U / c$ is the dimensionless time or the distance travelled in chord lengths, and $\boldsymbol{i}, \boldsymbol{k}$ are the unit vectors in the $x$ and $z$ directions, respectively. This definition of the foil's motion defines the point that the foil leaves the PIV plane as $t^{*}=0$. The programmed vertical motion of the foil is a smoothed S-curve generated by the linear motor software in order to produce the programmed motion step. The observed profile of the foil's vertical motion is given in figure 4 .

\subsection{Force measurements}

For a wing piercing a non-contaminated free surface (Slaouti \& Gerrard 1981) the effective aspect ratio can be approximated as double the physical aspect ratio of the wing for moderate Froude number:

$$
A R_{e f f}=\frac{2 b}{c},
$$

such that prior to pullout $A R_{\text {eff }}=12.5$; hence the flow can be approximated as twodimensional over a large spanwise region of the wing.

Measurements of the lift force on the wing as function of the angle of attack were performed, using an ATI industrial automation Gamma six-component force/torque sensor. The wing was towed for a distance of $1.8 \mathrm{~m}$ and the steady-state region of the lift force was averaged for repeated runs, for various angles of attack. Forces were sampled at $1000 \mathrm{~Hz}$ and the error in the lift coefficient based on the sensor sensitivity was $\Delta C_{l}= \pm 0.01$. The resulting lift measurements in figure 5 show that at this Reynolds number, the performance of the wing lies in between the results from 


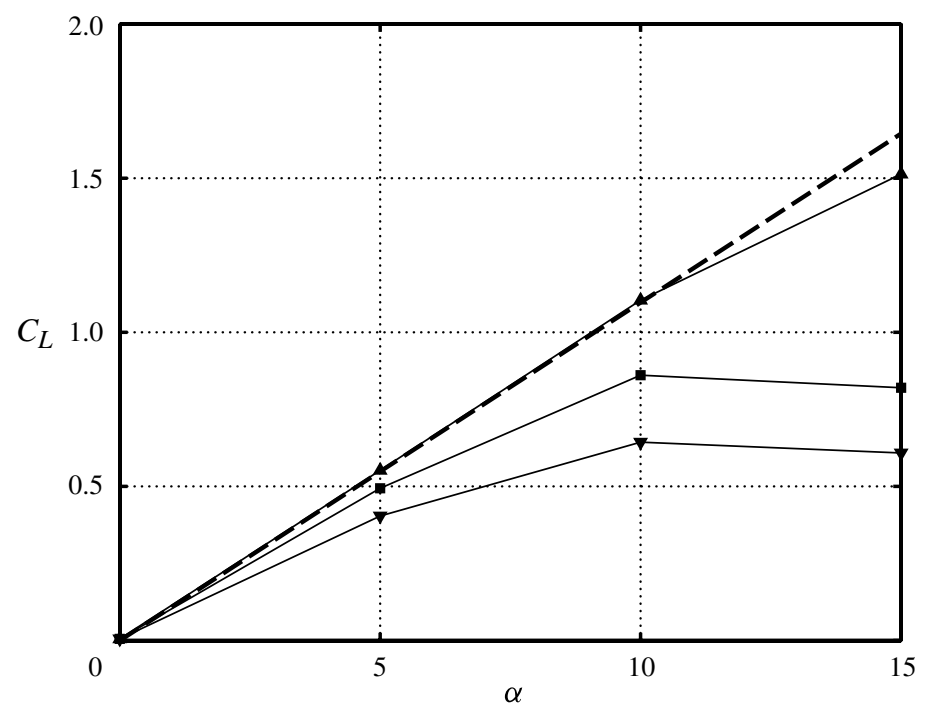

FIGURE 5. Measurement of the lift coefficient. Dashed line shows the theoretical lift coefficient from potential flow theory. $\mathbf{\square}$, current experiment at $R e=14000 ; \mathbf{\Delta}$, Abbott \& von Doenhoff (1959) at $R e=300000 ; \mathbf{\nabla}$, Alam et al. (2010) at $R e=10500$.

Abbott $\&$ von Doenhoff $(1959)(R e=300000)$ and Alam et al. $(2010)(R e=10500)$. The results suggest that at $\alpha=10^{\circ}$ stalling has commenced.

\subsection{Experimental flow visualization}

For quantitative flow visualization measurements, a time-resolved PIV system was used. The system was provided by LaVision $\mathrm{GmbH}$, consisting of a Quantronix Darwin Nd:YLF $(\lambda=527 \mathrm{~nm})$ single-cavity laser and a 10 bit Imager Pro HS CMOS camera. The camera recorded images in single-frame mode with a frame rate of $600 \mathrm{~Hz}$ at full resolution $(1280 \times 1024$ pixel $)$. The laser beam was collimated and then expanded into a sheet approximately $0.5 \mathrm{~mm}$ in thickness. For illumination, we used polyamid seeding particles with mean particle diameters on the order of $50 \mu \mathrm{m}$.

Two different setups in separate experiments were used for obtaining the velocity field using PIV. Both setups are illustrated in figure 6. In the first (figure $6 a$ ), the camera was mounted on the traverse, directly behind the foil. This setup allows visualization of the foil's wake, but the foil is not actually seen in the field of view. Since the foil is out of the field of view, the visualizations and calculations obtained through this method represent the state of the foil's wake well after the foil has vanished. In the second set of PIV measurements (figure $6 b$ ) the camera is stationary, located underneath the tank, while the foil is made to pass in front of the camera's field of view. In this setup, a more detailed description of the velocity field is obtained directly following the disappearance of the foil.

Vector fields were calculated using an adaptive correlation with $32 \times 32$ pixels interrogation windows and a $50 \%$ overlap. A local median filter was employed to eliminate outliers. A $3 \times 3$ median filter was also used to lightly smooth the vector fields in order to more clearly define the vortical structures in the wake. The accuracy of the vector fields was estimated to lie within $2 \%$ of the towing speed for all cases. Subsequently the calculation of vorticity and circulation could be estimated to have 
(a)

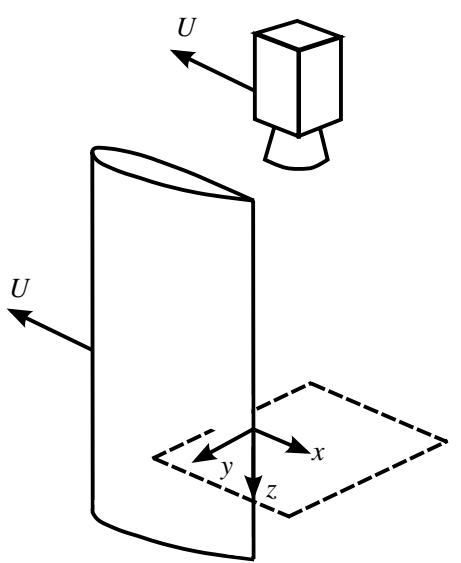

(b)

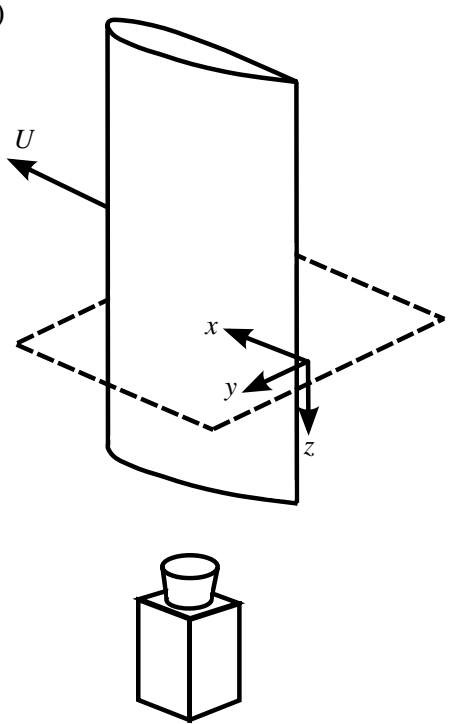

FIGURE 6. Schematics of the PIV system setup. (a) Setup 1: the camera is mounted on the moving carriage, viewing the wake behind the foil; hence the foil is never completely visible in this view. (b) Setup 2: the camera is at a fixed location under the tank and the foil vanishes from the laser plane after the foil enters the camera field of view.

uncertainties of $\Delta \omega c / U= \pm 0.1$ and $\Delta \Gamma / U c= \pm 0.01$, respectively. See Raffel et al. (2007) regarding the details of the error analysis.

The vorticity fields were calculated directly from the velocity vector fields using a centred-difference formulation. Circulation was subsequently calculated from the local vorticity field using Stoke's theorem via numerical integration:

$$
\Gamma=\iint_{A} \omega_{z} \mathrm{~d} A,
$$

where $A$ is the full measurement window in the wake of the wing. The net circulation was calculated as the sum of all circulation in the wake at a given moment in time, thus avoiding the challenge of arbitrarily defining vortex structures as described in Jeong \& Hussain (1995). In the second PIV setup, where the camera is stationary, circulation was calculated only for a bounding box around vorticity that was not in the wake of the foil prior to vanishing. In order to avoid vorticity from the foil wake entering the bounding box, the bounding box moves in time.

In images where the foil appears, the velocity field has been masked to avoid boundary effects near the surface of the foil. Once the foil has been removed from the PIV plane, masking is no longer necessary. Circulation calculations are only given for times $t^{*}>0$, after the foil has been removed from the PIV plane, since the foil's boundary layer cannot be resolved in the field of view prior to the removal of the foil.

\section{Vorticity in the near wake}

The near wake of the foil is visualized using PIV setup 1, where a moving frame follows the foil at the same speed as the foil's forward velocity. Figures 7-10 show the near wake of the foil at three instants in time after the foil has vanished, for different angles of attack. For comparison, the non-dimensional times shown for each angle of 

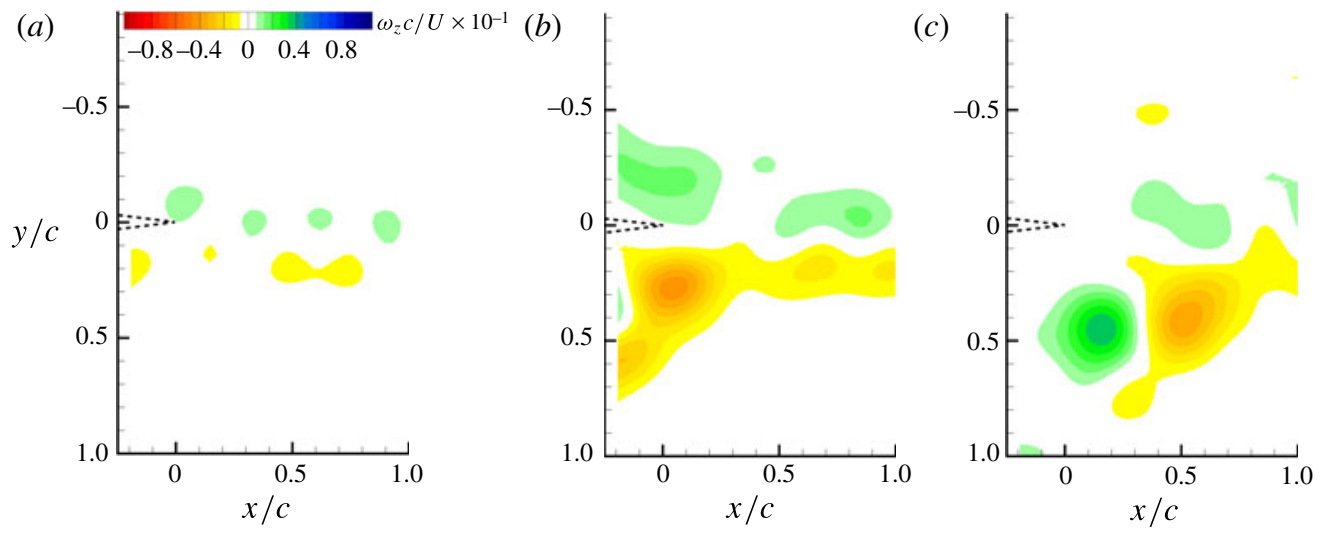

FIgURE 7. Dimensionless vorticity in the near wake of the vanished foil for $\alpha=0^{\circ}$, $t^{*}=0.09(a), t^{*}=0.34(b)$ and $t^{*}=0.59(c)$; note that the foil trailing edge is located at $x / c=0$ and $y / c=0$. Fluid flow is left to right.
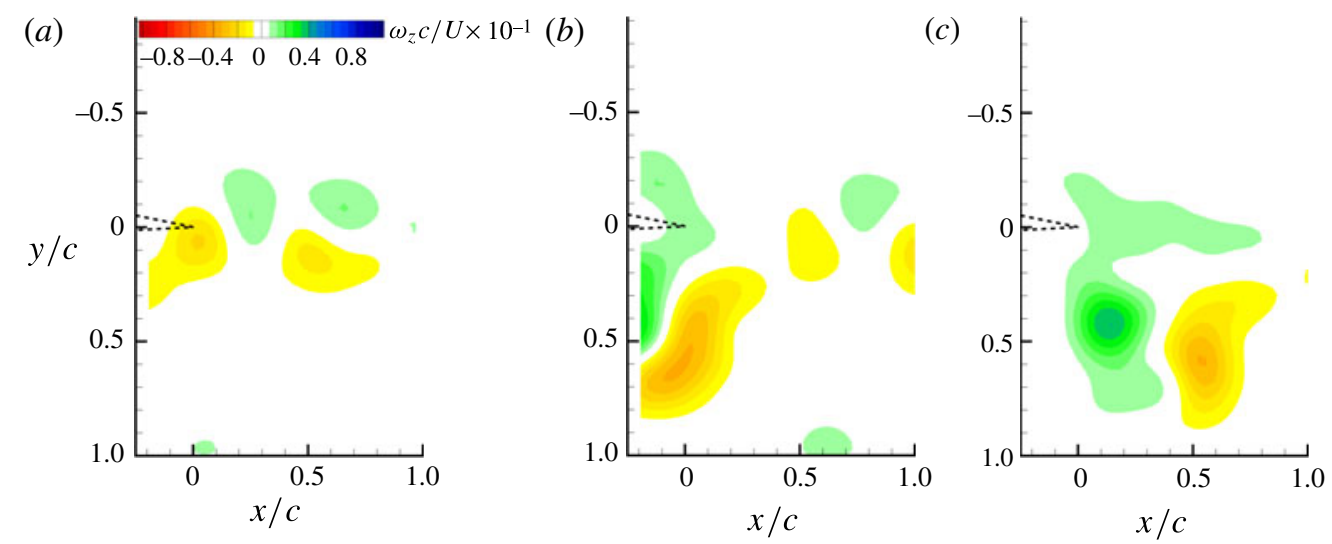

FIGURE 8. Dimensionless vorticity in the near wake of the vanished foil for $\alpha=5^{\circ}$, $t^{*}=0.09(a), t^{*}=0.34(b)$ and $t^{*}=0.59(c)$; note that the foil trailing edge is located at $x / c=0$ and $y / c=0$. Fluid flow is left to right.

attack are $t^{*}=0.09, t^{*}=0.34$, and $t^{*}=0.59$. Each visualization was repeated 10 times at each angle of attack; however, for brevity, only images from a single representative time series are shown.

The evolution of vorticity in the near wake of the foil for $\alpha=0^{\circ}$ is seen in figure 7 . At time $t^{*}=0.09$, the foil has been retracted from the PIV plane, but the camera is still looking in the wake downstream of the foil and only captures the Kármán street. At time $t^{*}=0.34$, the vorticity that emanates from the upper and lower boundary layers becomes visible in the form of two clear vortices on the left side of the image. By time step $t^{*}=0.59$ the resulting vortex pair is downstream of the cylinder and the induced velocity has moved the pair to one side of the field. It should be noted here that the vortex emanating from the lower side consistently leads the opposing vortex from the upper side, suggestive of a slight asymmetry in the wing.

In figure 8 , at $\alpha=5^{\circ}$, similar behaviour is observed to that of the $\alpha=0^{\circ}$ case; however, as expected, the resulting clockwise (positive) vortex at $t^{*}=0.59$ is stronger 

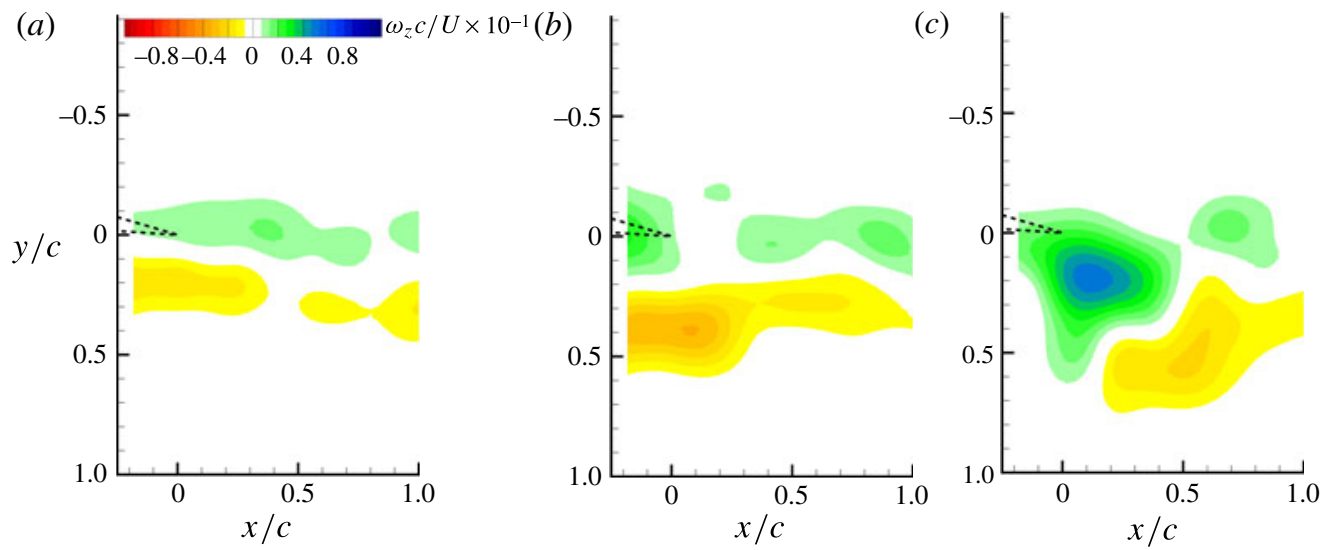

FIGURE 9. Dimensionless vorticity in the near wake of the vanished foil for $\alpha=10^{\circ}$, $t^{*}=0.09(a), t^{*}=0.34(b)$ and $t^{*}=0.59(c)$; note foil trailing edge located at $x / c=0$ and $y / c=0$. Fluid flow is left to right.
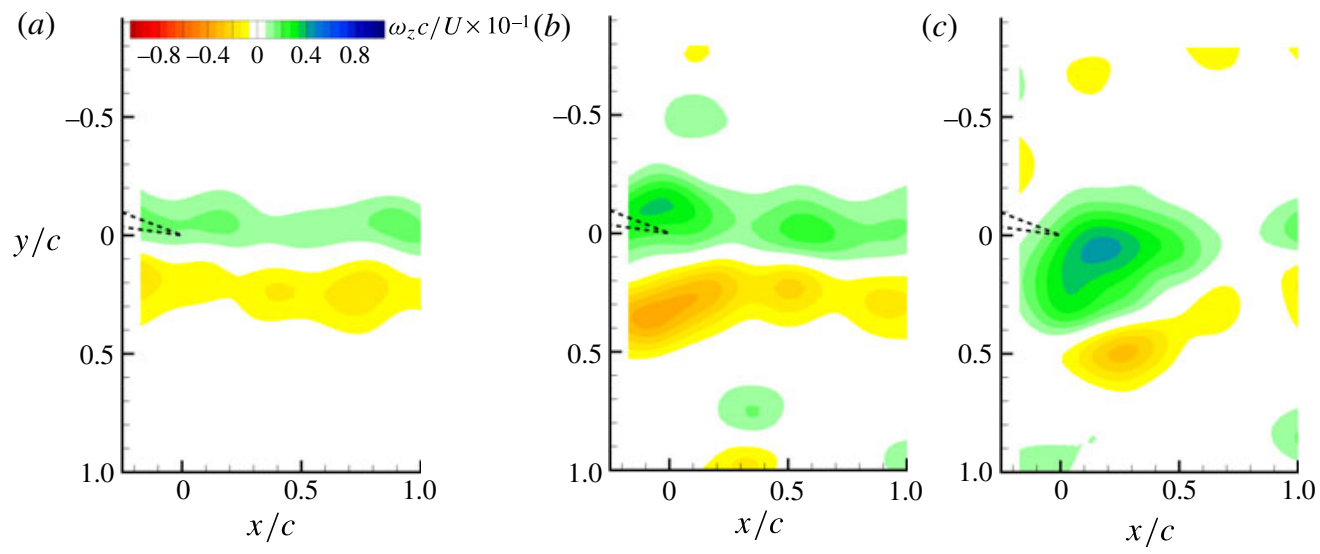

FIGURE 10. Dimensionless vorticity in the near wake of the vanished foil for $\alpha=15^{\circ}$, $t^{*}=0.09(a), t^{*}=0.34(b)$ and $t^{*}=0.59(c)$; note foil trailing edge located at $x / c=0$ and $y / c=0$. Fluid flow is left to right.

than the counterclockwise (negative) vortex, resulting in net positive circulation. Additionally, the final orientation of the vortex pair is slightly different, due to the angle of attack on the foil.

Similar results are also observed in figures 9 and 10 , for $\alpha=10^{\circ}$ and $\alpha=15^{\circ}$, respectively. The resulting clockwise vortices at $t^{*}=0.59$ are found to be progressively stronger and the orientation of the pair tends to line up parallel to the centreline of the foil. In these cases, the wake prior to the rapid upward displacement of the wing, as seen at $t^{*}=0.09$, contains two parallel shear layers indicative of stall. This stall was expected, as seen in the lift measurements from figure 5.

From the image sequence associated with figures $7-10$, it is possible to determine the circulation in the near wake of the vanished foil from (3.3). Figure 11 shows the resulting net circulation behind the vanishing wing, where $t^{*}=0$ is the point where the 


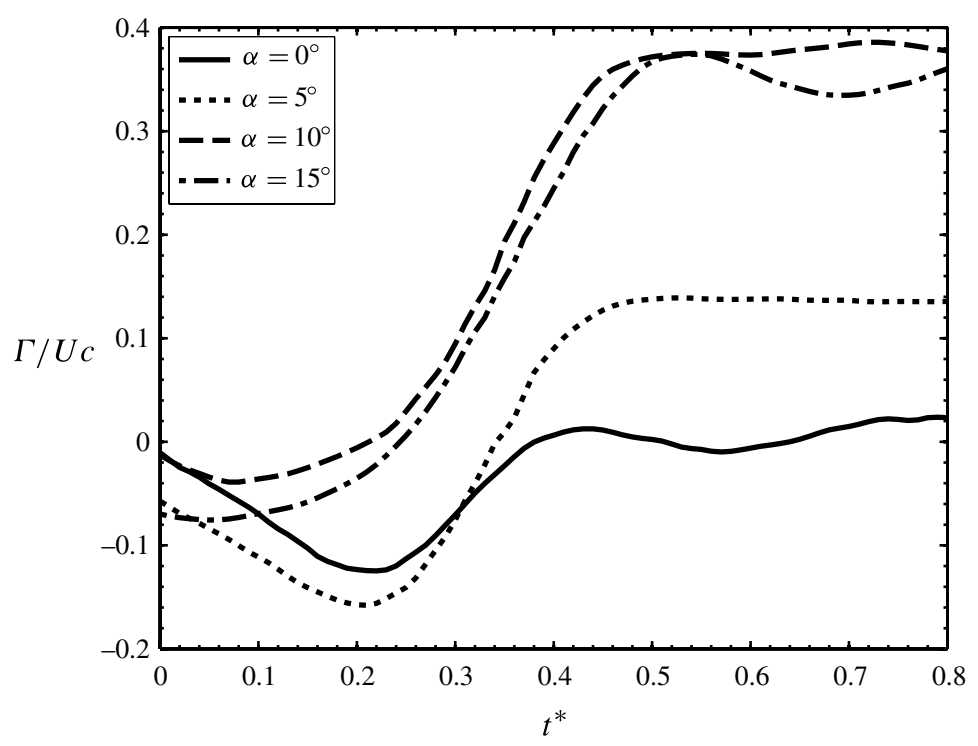

FIGURE 11. Net circulation measured in the wake of the vanishing wing using PIV where $t^{*}=0$ corresponds to the point where the foil passes through the PIV plane. Circulation is calculated as the average circulation from ten separate experiments at each angle of attack.

foil disappears from the laser plane. Since at time $t^{*}=0$, only the Kármán street is visible in the viewing field, the net circulation is near zero, depending on the phasing of the Kármán street formation. As time progresses, the vorticity associated with the foil's boundary layer enters the field of view, resulting in an increase in calculated circulation. Eventually, the value of circulation levels off at a maximum value which corresponds to the net circulation that existed on the foil before vanishing (bound circulation).

Figures 7-10 are shown for individual experimental runs. The experiments for each angle of attack were repeated 10 times each, in order to assess repeatability in the wake patterns. At each angle of attack, the resulting vortices in the wake were repeatable in both orientation and strength. Figure 12 shows the resulting vortex orientation for $\alpha=10^{\circ}$ and $t^{*}=0.59$ for three additional experiments. Along with the result shown in figure 9 , figure 12 shows that the basic orientation of the vortex pair is repeatable, with the centre of the vortices roughly oriented on a line parallel to the centreline of the foil. Additionally, the clockwise vorticity from the suction side is found to lead the counterclockwise vorticity from the pressure side in all cases. These observations hold despite the fact that there were unavoidable timing differences with respect to the Kármán street formation. In any case, the Kármán street vortices are much weaker than the vortices forming when the foil vanishes.

Applying Kutta-Joukowski's theory for a two-dimensional, thin, symmetric foil, one can estimate the net bound circulation on a slice through the wing as:

$$
\Gamma_{0}=\pi \alpha c U .
$$

At the relatively low Reynolds numbers in this study and for a finite-aspect-ratio wing, an experimental measurement of the lift force provides a better estimate of bound circulation. With these measured values of lift $(L)$, shown in figure 5, the estimate of 

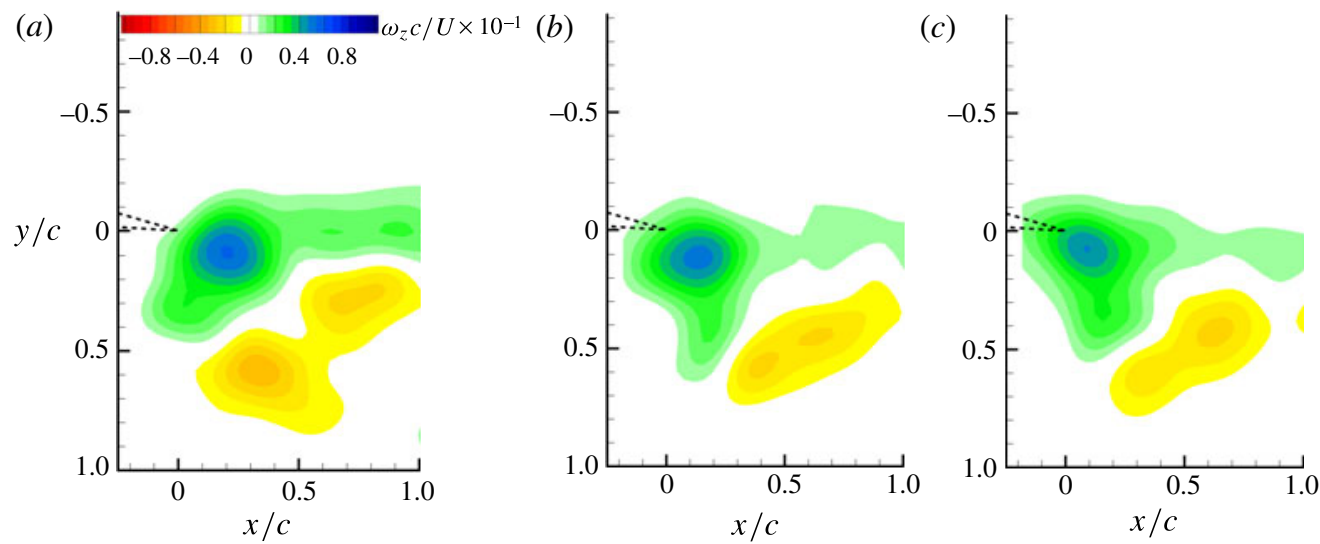

FIGURE 12. Dimensionless vorticity in the near wake of the vanished foil for $\alpha=10^{\circ}$ and $t^{*}=0.59$ for three additional experiments. Note that the foil trailing edge is located at $x / c=0$ and $y / c=0$. Fluid flow is left to right. The basic orientation of the vortex pair emanating from the boundary layers is repeatable despite differences in the phasing of the Kármán wake downstream.

the net bound circulation is:

$$
\Gamma_{0}=\frac{L}{\rho U} .
$$

In figure 13 a comparison is made between the resulting bound circulation, determined through (4.2) using the force balance, and the net circulation measured in the wake from PIV. Good agreement between these measurements is observed, showing that the original bound vorticity on the wing is conserved when transferred into the wake. This also confirms that possible three-dimensional effects from the foil passing through the laser plane are negligible for large $t^{*}$ as three-dimensional effects would influence the calculation of vectors in the two-dimensional PIV plane. Note that the error bars associated with the wake measurements represent one standard deviation, obtained from ten separate runs obtained with PIV setup 1. The circulation obtained through the direct force measurements are accurate to within $\Delta \Gamma / U c= \pm 0.01$.

The timing of the transfer of vorticity to the wake from the boundary layer is difficult to assess quantitatively with this camera arrangement, since vorticity advects into the PIV viewing field slowly in time. In order to fully understand the process by which vorticity is rapidly transferred from the foil's boundary layer into the fluid, it is necessary to visualize the vanishing process directly. This is achieved through a repositioning of the viewing window, as explained in the next section.

\section{The reconfiguration of vorticity as the foil vanishes}

In order to explore the reconfiguration of the boundary layer vorticity after it is shed from the vanishing foil, PIV setup 2 is used, where the camera is placed at a fixed location underneath the tank and the foil passes in front of the camera. Optical switches are used to trigger the camera and the foil motion, such that the experiment is repeatable and the foil vanishing scenario occurs at the same point in time and space with respect to the camera images. The experiment is repeated 30 times at a $10^{\circ}$ angle of attack, such that an ensemble average of the velocity field can be attained. 


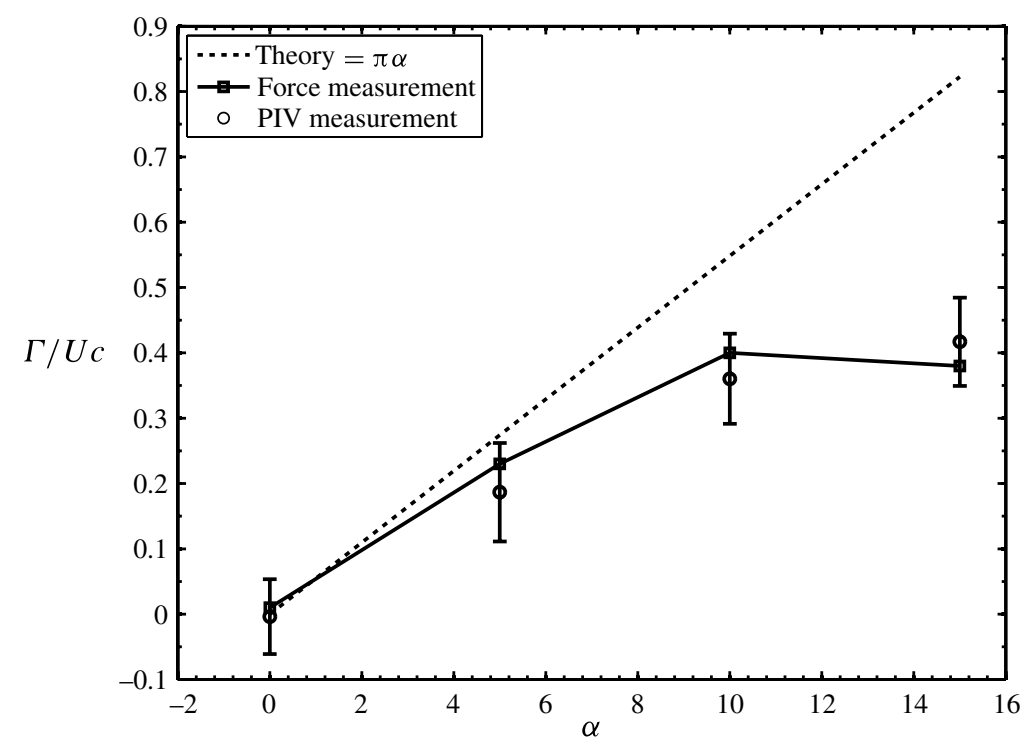

FIGURE 13. Comparison between the circulation derived from the measured lift before wing removal (equivalent to figure 5) and that obtained from the wake after wing removal, using PIV. The error bars associated with the wake measurements represent one standard deviation calculated from ten experimental runs.

The averaged vorticity field derived from the velocity field is shown in figure 14 . The velocity field is masked for images in which the foil is still present and a boundary region around the foil is also masked to avoid boundary effects on the calculation of vorticity.

Figure 14 shows a detailed sequence of images from time $t^{*}=-0.01$ to $t^{*}=0.08$, and then a coarser sequence of images up to $t^{*}=1.00$. At negative values of $t^{*}$, the foil has not yet vanished from the field of view and the boundary layer is still attached to the foil. At the instant of vanishing, defined as $t^{*}=0$, the foil has just exited the plane of view and two, clearly seen, free shear layers are present. The shear layers begin immediately to roll up, resulting in two strong vortices, labelled A and B, which have completely formed by time $t^{*}=0.03$. The negative-sign vortex $\mathrm{B}$ (clockwise vorticity coming from the upper part of the foil) is positioned forward of positive-sign vortex A. Other smaller vortices form as well, but the two dominant vortices remain stable; no complex motions characteristic of multi-vortex arrangements (Aref 1983) are observed.

At time $t^{*}=0.05$, two new vortices appear in the plane, labelled $\mathrm{C}$ (positive sign) and D (negative sign), positioned between the two previously formed vortices A and B. These secondary vortices grow in intensity until they are fully formed around $t^{*}=0.08$. After time $t^{*}=0.08$, and as time progresses, the secondary vortices $\mathrm{C}$ and $\mathrm{D}$ weaken and appear to ultimately coalesce with the originally formed vortices $\mathrm{A}$ and $\mathrm{B}$. The resulting combined vortices remain in the wake past $t^{*}=1.00$.

As we show in the next section, vortices $\mathrm{C}$ and $\mathrm{D}$ are the result of three-dimensional structures, which form due to a vortex ring developing at the sharp-edged lower end of the foil as it is pulled up. The ring entrains flow and vorticity with it. Vortices $\mathrm{C}$ and $\mathrm{D}$ connect this ring with vortices $\mathrm{A}$ and $\mathrm{B}$, as well as with the original tip vortex.

The resulting vortex pair at $t^{*}=1.00$ is the same pair seen in figure 12 . Figure 15 shows the total positive vorticity, total negative vorticity, and total net circulation as 


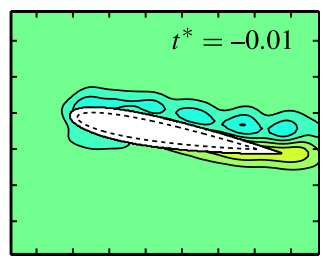

Foil vanishes, boundary layers
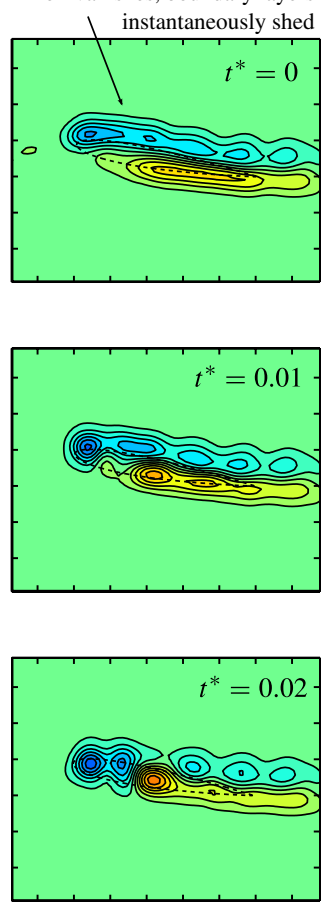

Formation of strong vortices

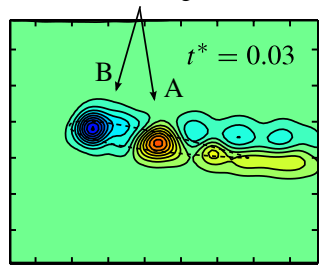

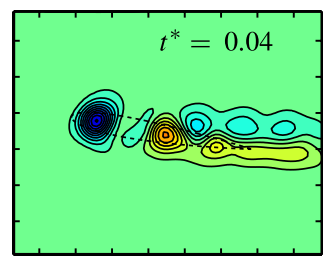

Appearance of secondary vortices
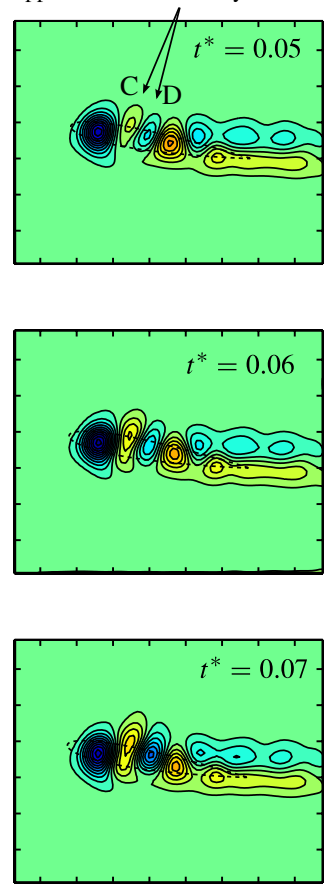

Evolution of strong and secondary vortices

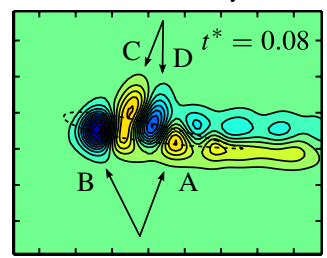

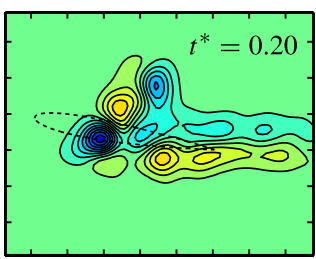
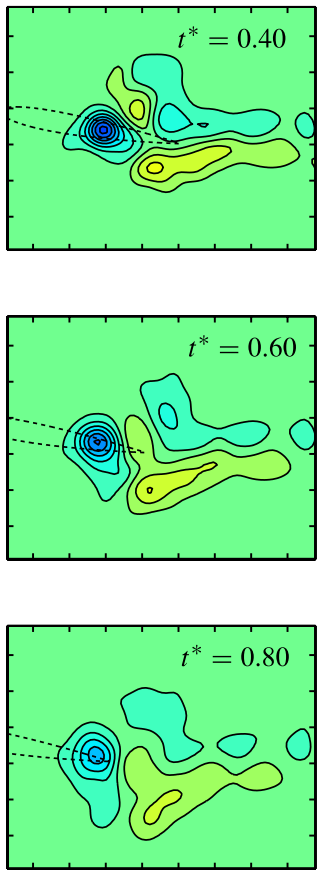

Lasting vortices in wake

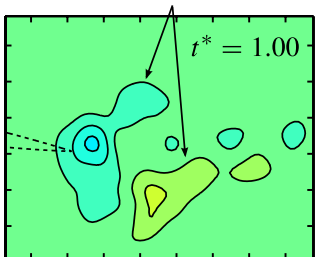

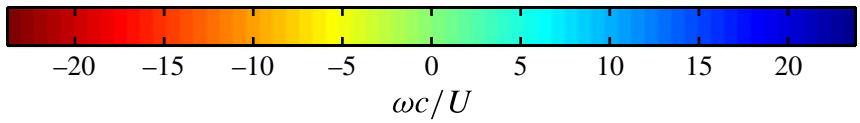

FIGURE 14. Vorticity field for foil at $10^{\circ}$ angle of attack with varying $t^{*}$. The foil appears to vanish and forms two free shear layers of unequal circulation at $t^{*}=0$, which then curl up into two vortices, $\mathrm{A}$ and $\mathrm{B}$. Vortices $\mathrm{C}$ and $\mathrm{D}$ are caused by three-dimensional effects from the foil passing through the PIV plane. Time steps follow from top to bottom and left to right. Shown time steps are $t^{*}=-0.01,0,0.01,0.02,0.03,0.04,0.05,0.06,0.07,0.08,0.2,0.4,0.6,0.8,1.0$. The foil is moving right to left in a stationary fluid. Contours of non-dimensional vorticity show levels of $\omega_{z} c / U \pm 2.1$. Tick marks show a spacing of 0.2 chord lengths. 


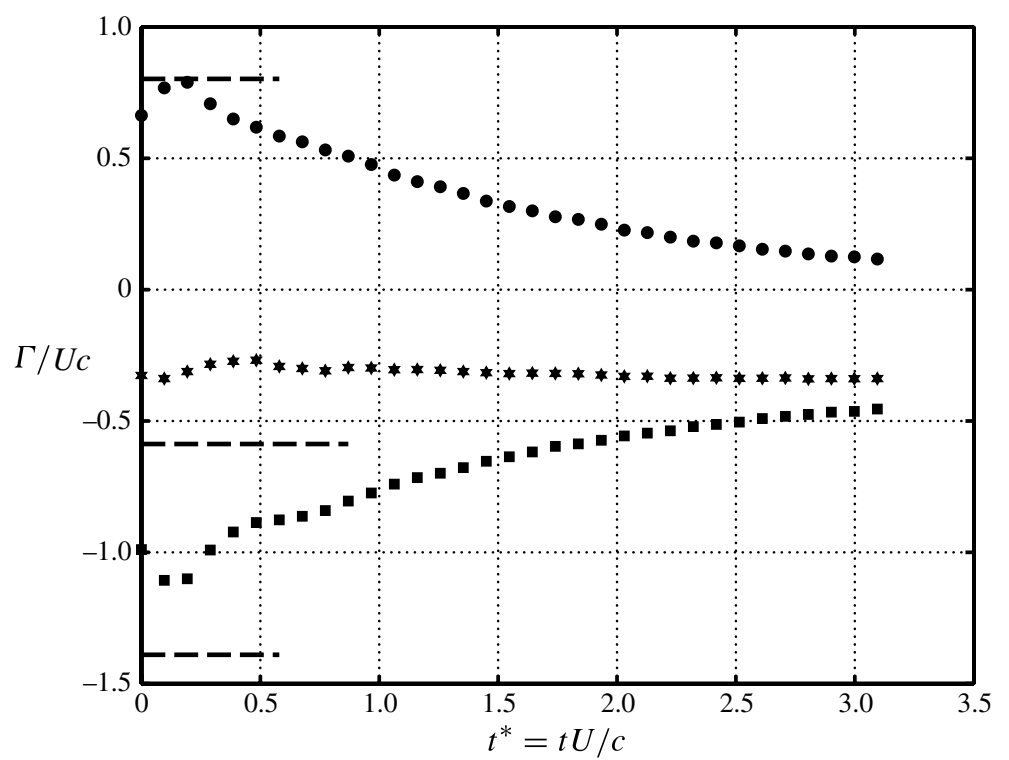

FIGURE 15. Circulation calculated from PIV images for the free vorticity after the foil has vanished. Calculation is done over a bounding box that moves at the rate of the foil wake and encompasses only vorticity from the foil's shed boundary layer. Calculations are given for the net circulation $(\star)$ along with only positive $(\bullet)$ and only negative $(\boldsymbol{\square})$ circulation. Circulation derived from potential flow for a Joukowski foil with similar geometry to NACA0012 is also given for reference as the dashed lines.

functions of time. The initial increase in negative and positive vorticity is the result of the appearance of vortices $\mathrm{C}$ and D. Vorticity then decays as a result of annihilation of positive and negative vorticity (Morton 1984), as well as the stretching of vortices $\mathrm{C}$ and $\mathrm{D}$, but the net circulation remains approximately constant. In figure 15 , the net circulation in the vorticity field is calculated within a bounding box that encompasses the shear-layer vorticity after $t^{*}=0$, excluding the Kármán-street vorticity previously shed from the foil (Zdravkovich 2003). The calculation bounding box moves in time $t^{*}$ with the velocity of the Kármán street in order to avoid including vorticity from the foil's wake.

Figure 15 shows the estimates of shed vorticity from the upper side (negative vorticity) and lower side (positive vorticity) of the foil, as well as the bound circulation, based on Joukowski's two-dimensional foil theory, i.e. without accounting for finite-span and Reynolds-number effects. Around the time that the foil is about to vanish, the peak positive and negative circulations in the field are close to the theoretical values. As time progresses, the positive and negative circulation in the field decreases, however the net circulation remains approximately constant. Agreement is good given the simplifying assumptions used in the theoretical calculations. The net circulation does fluctuate slightly due to the three-dimensionality of the flow; however, over longer time, the measured net circulation in the plane is equal to the initial value found directly after the foil vanishes. The steady value of the net circulation for large $t^{*}$ indicates that three-dimensional effects are largely confined to times near $t^{*}=0$.

There is a small growth in vorticity evident in figure 15 for $t^{*}<0.2$. Figure 16 shows a more detailed calculation of circulation over this short time period. The circulation for each individual vortex is calculated by integrating vorticity within 

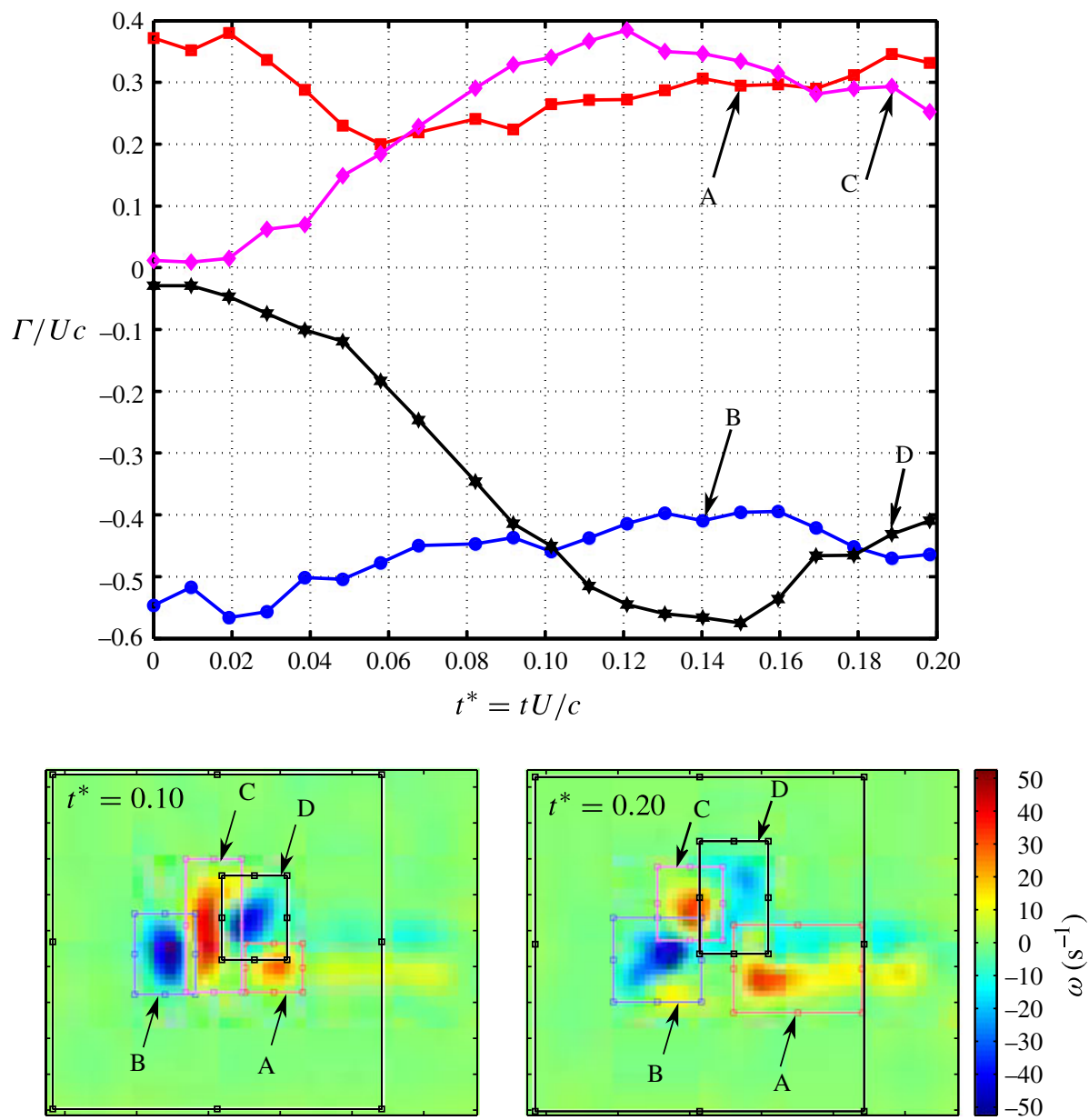

FIGURE 16. Individual calculation of circulation for labelled vortices A, B, C and D for times $t^{*}$ between 0 and 0.2 . Coloured boxes on the vorticity field plots below show the area of vorticity integration for calculation of circulation. $\mathbf{\square}$, vortex A (red); •, vortex B (blue); $\diamond$, vortex C (pink); $\star$, vortex D (black). Multiple boxes are used to more finely isolate individual vortex structures. Primary vortices A and B slowly decay in strength, while secondary vortices $\mathrm{C}$ and $\mathrm{D}$ appear to grow over a short period of time and then begin to decay as well.

a bounded region around each vortex. Several bounding boxes are used to define the region of integration for each vortex as shown in sample images illustrating the calculation (see figure 16). Within each bounding box, only positive or negative vorticity is integrated depending on the sign of the particular vortex of interest. The strength of vortices $\mathrm{A}, \mathrm{B}, \mathrm{C}$ and $\mathrm{D}$ is shown as a function of non-dimensional time. The primary vortices $A$ and $B$ start at a certain value of circulation; this value decreases slowly in time. The initial values of circulation for A and B differ from the total positive and negative circulations shown in figure 15, since a major portion, but not the entire vorticity, from each shear layer rolls into vortices A and B. Vortices $\mathrm{C}$ and $\mathrm{D}$ grow in time as they enter the viewing plane due to three-dimensional flow; hence, vortices $\mathrm{C}$ and $\mathrm{D}$ are not initially present. These vortices rapidly grow in strength reaching a value close to that of vortices A and B, and finally decay. 


\section{Three-dimensional vortex structures}

The foil in this experiment does not 'vanish', but rather is pulled upwards and outside the observation plane. In contrast to the thought experiment of Taylor (1953), where the vanishing disk is assumed to instantly change to fluid form (much like instantaneously melting ice), the movement of the foil in the vertical direction creates a tilted upwards flow, following the motion of the foil. The lower tip of the foil has sharp edges, hence it feeds additional shear layers that curl up to form a ring-like vortex structure directly underneath the foil, lying roughly on a horizontal plane. This ring-like vortex, combined with the tip vortex shed by the free end of the foil create a three-dimensional fluid flow near the tip, which we analysed quantitatively using numerical simulation.

For the three-dimensional simulation of the foil being rapidly pulled upward in a steady streaming fluid we used the same foil geometry as in the previous experiments. The angle of attack is set to $10^{\circ}$ and the vertical position of the foil is prescribed to change smoothly but rapidly, with an average speed three times that of the forward motion, as in the experiments. A robust immersed boundary method suitable for dynamic non-deformable bodies is used to simulate the flow around the foil. The numerical details of the simulation method follow those used in Weymouth et al. (2006) and Weymouth \& Yue (2011). The Reynolds number, based on the forward speed and the chord length, is set to 14000 , as in the experiments. The effect of turbulence is modelled using an implicit large-eddy simulation (ILES) scheme (Margolin, Rider \& Grinstein 2007), which preserves the time-varying structures in the wake. More than $3.1 \mathrm{M}$ grid points are used, with 50 points along the chord length, to ensure resolution of the small-scale features. Vortex cores in the resulting flow are visualized using iso-surfaces of the $\lambda_{2}$ metric of Jeong \& Hussain (1995). The vortex core surfaces are then coloured using contours of the $z$-axis component of the vorticity in order to illustrate the rotation of the vortex cores. A schematic drawing of the vortex structures connecting to the foil is shown in figure 17, while visualizations from the numerical simulation are shown in figure 18. Based on Prandtl's lifting-line theory and the tight bundling of vortex filaments in the near wake of a rectangular wing, as found in the near-wake study by Birch \& Lee (2005), the tip-vortex circulation has equal strength to that of the bound vortex. As the foil moves forward at a constant angle of attack, before being pulled up, the lines of circulation on each side of the foil (labelled A and B in figure 17) will simply connect at the tip of the foil, with the tip vortex (labelled T) indicating the sum circulation from the pressure and suction sides. When the foil is rapidly pulled upwards, as in the position shown in figure 17, the connection of circulation lines breaks at the tip vortex and a ring-like vortex structure forms (labelled R) on the underside of the foil, due to the additional upwards motion of the foil. The break points at the tip vortex result in additional vortex structures $\mathrm{C}$ and $\mathrm{D}$ that connect the ring vortex to the tip vortex.

Figure 18 shows the vortex core connections as found in the simulation. The bound circulation lines represented by A and B are not initially vortices. Instead, they consist of the vorticity of the boundary layer of the foil that is then released in the form of a free shear layer in the fluid as the foil moves upward. The $\lambda_{2}$ metric of Jeong \& Hussain (1995) illustrates regions where the rotation of the flow is high relative to its translation; hence, the free shear layers will not immediately appear as a vortex core in the simulation. From figure 18, we see two instants in time as the foil is undergoing its upward motion. At $t^{*}=0.05$, the tip vortex has bent upward, a ring 


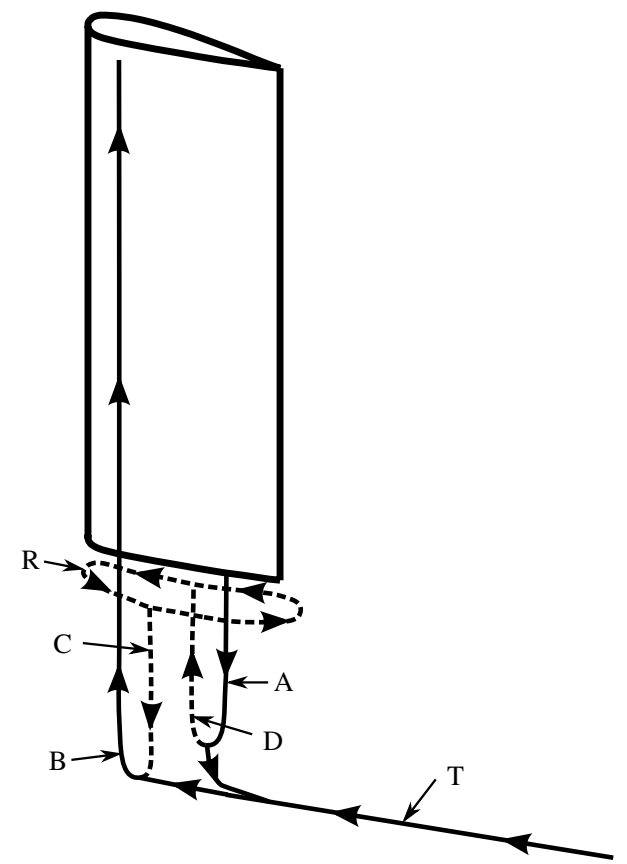

FIGURE 17. Schematic of three-dimensional vortex topology as the foil is pulled upward. Letter labels show the primary vortex structures formed in this motion, as described in the text.

vortex has formed beneath the foil tip, and the connection between the tip vortex and bound vorticity is beginning to split apart. Vortices A and B are visible at the splitting point, while vortices $\mathrm{C}$ and $\mathrm{D}$ are not yet fully formed. At $t^{*}=0.13$, the vortex core connections between the tip vortex and the foil are more clearly seen. In this image, the limitation of the vortex core visualization is apparent as vortices A and B must connect to the boundary layer of the foil, which is not visible as a contour of $\lambda_{2}$.

As the foil moves upward, the free shear layers left in the fluid collapse to form vortex cores. The formation of a vortex ring underneath the foil forces the shed free layers to wrap around and entrains part of the shed vorticity upwards, because the ring follows the upward motion of the foil. This results in the formation of vortices labelled $\mathrm{C}$ and D, which connect to the ring and are continuously stretched as the foil is pulled upwards. The schematic in figure 17 shows the resulting idealized topology; however, as seen in figure 18, there are additional small core structures present that reconnect the tip vortex to the ring. For instance, at $t^{*}=0.13$, one can see that vortex $\mathrm{C}$ consists of two cores connecting to the tip vortex. This variation in the formation of vortices was apparent in individual runs from experiments as well. As time progresses, these vortex structures interact with one another as the wake evolves in time. However, the two initial vortices, $\mathrm{A}$ and $\mathrm{B}$, dominate the structure of the wake and are persistent for a long time after the foil has moved upwards. The dominance of vortices A and $\mathrm{B}$ is evident in figure 18 at $t^{*}=0.25$. At this point in time, two strong vortex tubes are clearly formed, aligned in the spanwise direction of the foil. The alignment and strength of vortices $\mathrm{A}$ and $\mathrm{B}$ indicate that at this later $t^{*}$, the resulting flow is largely two-dimensional and the three-dimensional effects from the foil tip are not significantly affecting the flow at the midpoint of the foil's spanwise excursion. 

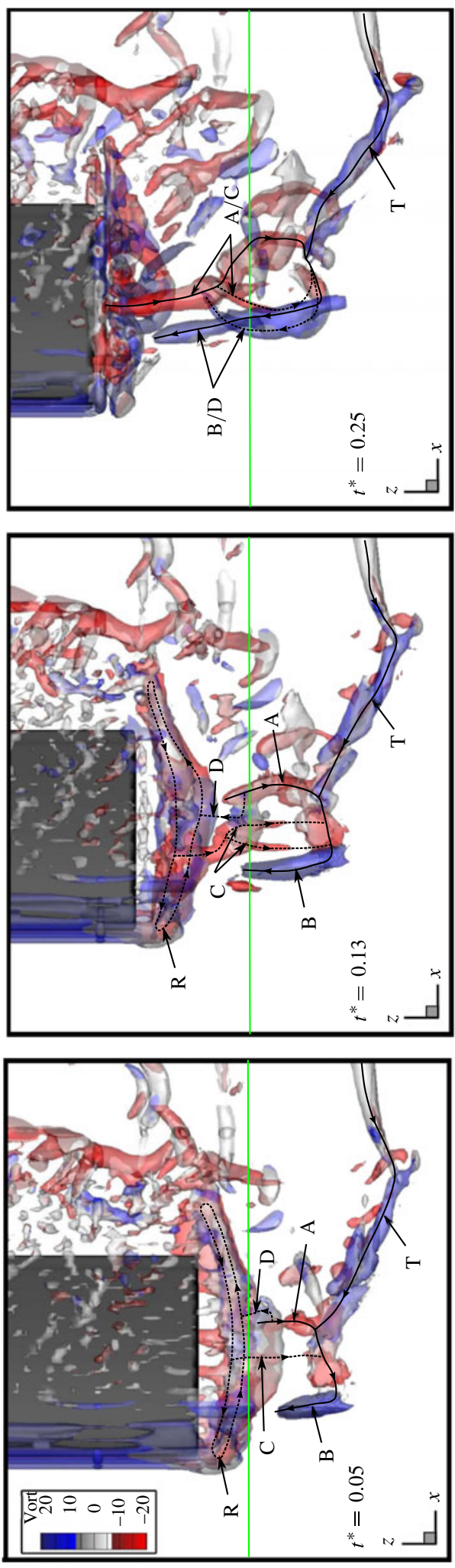

远 总

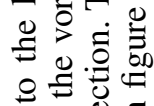
bo 8.

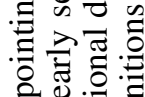

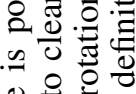

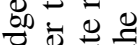
on 0 。 $\exists . \Xi . \Xi$ on $\Phi$ 으의 궁 氝 웅 후웜 웡

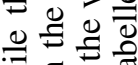
的 든 च 해용 ․ㅡㄹ. 过司宁

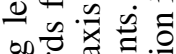
今 记

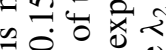

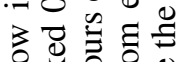

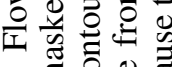
的 웧

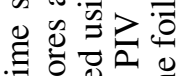

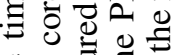
\&

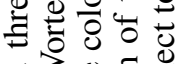
궁

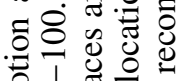

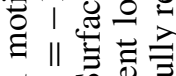

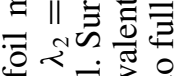

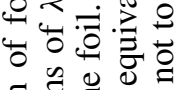
흐웛

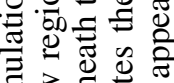

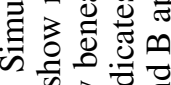
$\infty$ क त. - o $0<$

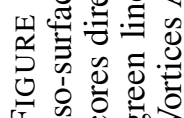




\section{Discussion}

The main conclusion from this analysis is that a rapidly retracted foil causes the formation of two strong spanwise vortices through the mechanism of global vorticity shedding. One vortex forms from the high-pressure side of the foil and the other forms from the low-pressure side. Each vortex contains a major part of the circulation in the boundary layer from which it was generated. Three-dimensional effects at the tip of the foil have been documented in order to clarify the reconnection of the spanwise vortices with previously shed vortices, in particular the tip vortex and Kármán street vortices. Figure 18 illustrates the detailed reconnection information, showing the formation of the strong spanwise vortices.

The rapid transfer of the boundary-layer vorticity to the wake provides a mechanism for rapid vortex formation within the fluid that may be utilized for flow control. This study has focused primarily on the effect of varying the angle of attack on the wake features, while keeping the foil motion the same. A more detailed study will be necessary to characterize the effect of variations in the pull-up speed, pull-up travel distance, and foil end conditions, which may significantly affect the formation of vortices in the wake.

Although this study has focused primarily on the fluid structures associated with this simple foil motion, the repeatable orientation and predictable circulation of the resulting vortices forming in the free fluid means that they could be used as a tool for force generation or flow control. In this scenario, the time associated with the rapid transfer of vorticity into the free fluid becomes an important parameter. We have already defined the relevant time parameter governing this process as $t^{*}$. The definition of $t^{*}$ is similar to the definition of formation number discussed by Dabiri (2009). However, the formation time of Dabiri (2009) is defined for a system where vorticity is fed into the fluid through either point sources in two dimensions, or a closed line in three dimensions, rather than having a (nearly) instantaneous transfer of boundary layer vorticity into the free fluid. Here the time is non-dimensionalized by the forward velocity and the chord length, which is reasonable because the chord defines the characteristic length scale of the entire shear layer that is left in the fluid after the foil is removed.

In figure 14, we see that the formation of two strong vortices from the free shear layer occurs near $t^{*}=0.03$; hence for $\alpha=10^{\circ}$, we have an extremely fast transition from free shear layers to completely formed vortices. These vortices do not last long at peak strength due to three-dimensionality in the flow. As some vorticity is annihilated, the system stabilizes and like-signed vorticity coalesces. The two vortices seen in figures $7-10$ do not materialize until $t^{*} \approx 0.4$. Therefore, these weaker vortices left in the free fluid require an order of magnitude longer time to develop than the originally formed vortices.

As already discussed, in addition to having a predictable orientation and strength, the redistribution of vorticity in the fluid occurs over a very short period of time. A clear advantage to this method of vortex generation is the relative low power required to achieve such a move. In contrast to a control surface that changes angle of attack or flaps in the fluid as in Buchholz \& Smits $(2006,2008)$, and von Ellenrieder et al. (2003), this wing moves spanwise to the flow. With flapping motion, a substantial amount of fluid must be accelerated along with the flapping body in order to release vorticity into the fluid. In contrast, the rapid spanwise motion of the foil is achieved with relatively little acceleration of the surrounding fluid, meaning that the mass of the wing itself is the critical parameter in energy expenditure for this type of motion. 
The interaction of a free vortex in a fluid with a body can be used as a flow control method for achieving desired forcing on a body (Rockwell 1998). Once free vortices have formed, after the removal of the wing, they can act on another nearby body to cause forces that may be used to advantage. For example, suction forces could be utilized for rapid manoeuvring control, wherein the magnitude of circulation of vortices in the fluid is critical to inducing a large force on an object.

If we consider the pressure associated with the potential for a point vortex, then the pressure field for this vortex is

$$
p-p_{\infty}=-\rho \frac{|\Gamma|^{2}}{2(2 \pi R)^{2}} .
$$

In (7.1), $p-p_{\infty}$ is the local pressure relative to the pressure at infinity, $\rho$ is the density of the fluid, $\Gamma$ is the circulation of the vortex, and $R$ is the radius of the vortex core. From this equation, we find that as either the strength of the vortex decreases or the radius of the vortex increases, the minimum pressure will decrease rapidly. We have shown that the magnitude of circulation is largest immediately after the foil has been removed $\left(t^{*}=0.03\right)$ and the free shear layers have quickly collapsed into two strong vortices. Additionally, at this point, the vortex core is tightly wound; hence the radius of the vortex is small and there will be a large suction pressure at the core. In contrast, at $t^{*}=1$, although two vortices still persist in the wake, the useful suction pressure available is greatly reduced as the strength of individual vortices has decreased and the radius of the vortices has increased. Therefore, to most effectively use the forming structures, it would require bringing the forming vortices in contact with another body quickly.

\section{Conclusions}

We show that for a foil moving at constant angle of attack and steady velocity $U$, rapid removal of the foil in the spanwise direction results in a nearly-instantaneous transfer of vorticity from the boundary layer of the body to the fluid in the form of free shear layers, which quickly transition to form two strong, unequal-strength vortices that persist in the fluid for a significant time. We demonstrate this by rapidly pulling a surface-piercing foil out of the water, accelerating it in the spanwise direction while it is being towed steadily at constant angle of attack. The shed boundarylayer vorticity is shown to rapidly form two strong spanwise vortices with net total circulation equal to the bound vorticity. The orientation of this vortex pair is such that the vortex associated with suction-side vorticity will always be forward, while a line drawn through the centres of the two vortices will be roughly parallel to the centreline of the foil. Although this process is three-dimensional near the tip of the foil, the circulation of each of the forming vortices remains close to the original vorticity of the boundary layer, demonstrating that three-dimensional effects have small influence on these vortices.

The transition of the boundary-layer vorticity to form two strong vortices, marked as vortices $\mathrm{A}$ and $\mathrm{B}$ in the figures of this paper, occurs over a very short non-dimensional time, roughly over $t^{*}=0.03$, based on the steady towing speed and the foil chord. Additional vorticity is shed at the lower sharp-edged end of the foil in the form of a vortex ring which is connected to the previously shed tip vortex of the foil, as well as vortices $\mathrm{A}$ and $\mathrm{B}$. These connecting vortices interact with vortices $\mathrm{A}$ and $\mathrm{B}$, but are stretched as the vortex ring is pulled away, following the axially-pulled foil. As a result, over a longer period of time, $t^{*} \approx 0.4$, vortices $\mathrm{A}$ and $\mathrm{B}$ dominate the field. 
The repeatability, short time scale of vorticity transfer, and considerable strength of the forming vortices make this phenomenon potentially useful for flow control applications. Improvements to tip shape and foil movement could potentially reduce three-dimensional effects and improve vorticity transfer for specific flow control applications.

\section{Acknowledgements}

The authors wish to acknowledge support from the Singapore-MIT Alliance for Research and Technology, and from the MIT Sea Grant Program.

\section{REFERENCES}

Aвbott, I. H. \& von Doenhoff, A. E. 1959 Theory of Wing Sections. Dover.

Alam, Md. M., Zhou, Y., Yang, H. X., GuO, H. \& Mi, J. 2010 The ultra-low Reynolds number airfoil wake. Exp. Fluids 48, 81-103.

AREF, H. 1983 Integrable, chaotic and turbulent vortex motion in two-dimensional flows. Annu. Rev. Fluid Mech. 15, 345-389.

BetZ, A. 1950 Wie Entsteht ein Wirbel in einer Wenig Zähen Flüssigkeit? Die Naturwissenschaft 37, 193-196.

BIRCH, D. \& LEE, T. 2005 Investigation of the near-field tip vortex behind an oscillating wing. J. Fluid Mech. 544, 201-241.

Blondeaux, P., Fornarelli, F., Guglielmini, L., Triantafyllou, M. S. \& Verzicco, R. 2005 Numerical experiments on flapping foils mimicking fish-like locomotion. Phys. Fluids 17, 113601.

Buchiolz, J. H. J. \& Smits, A. J. 2006 On the evolution of the wake structure produced by a low-aspect-ratio pitching panel. J. Fluid Mech. 546, 433-443.

Buchrolz, J. H. J. \& Smits, A. J. 2008 The wake structure and thrust performance of a rigid low-aspect-ratio pitching panel. J. Fluid Mech. 603, 331-365.

Childress, S., Vandenberghe, N. \& Zhang, J. 2006 Hovering of a passive body in an oscillating airflow. Phys. Fluids 18, 117103.

DABIRI, J. O. 2009 Optimal vortex formation as a unifying principle in biological propulsion. Annu. Rev. Fluid Mech. 41, 17-33.

Dickinson, M. 2003 Animal locomotion: how to walk on water. Nature 424, 621-622.

Dong, H., Bozkurttas, M., Mittal, R., Madden, P. \& Lauder, G. V. 2010 Computational modelling and analysis of the hydrodynamics of a highly deformable fish pectoral fin. J. Fluid Mech. 645, 345-373.

von Ellenrieder, K. D., Parker, K. \& Soria, J. 2003 Flow structures behind a heaving and pitching finite-span wing. J. Fluid Mech. 490, 129-138.

Hsieh, S. T. \& LAUder, G. V. 2004 Running on water: three-dimensional force generation by basilisk lizards. Proc. Natl Acad. Sci. USA 101, 16787.

Hu, D. L. \& Bush, J. W. M. 2010 The hydrodynamics of water-walking arthropods. J. Fluid Mech. 644, 5-33.

Hubel, T. Y., Hristov, N. I., Schwartz, S. M. \& Breuer, K. S. 2009 Time-resolved wake structure and kinematics of bat flight. Exp. Fluids 46, 933-943.

Jeong, J. \& Hussain, F. 1995 On the identification of a vortex. J. Fluid Mech. 285, 69-94.

Johansson, L. C. \& Norberg, R. A. 2003 Delta-wing function of webbed feet gives hydrodynamic lift for swimming propulsion in birds. Nature 424, 65-68.

Johansson, L. C. \& Norberg, U. M. L. 2001 Lift-based paddling in diving grebe. J. Expl Biol. 204, 1687-1696.

KADEN, H. 1931 Aufwicklung einer unstabilen Unstetigkeitsfläche. Ing.-Arch. 2, 140-168.

KLEIN, F. 1910 Über die Bildung von Wirbeln in reibungslosen Flüssigkeiten. Z. Math. Phys. 58, 259-262. 
Lentink, D., Müller, U. K., Stamhuis, E. J., De Kat, R., Van Gestel, W., Veldhuis, L. L. M., Henningsson, P., Hedenström, A., Videler, J. J. \& VAn Leeuwen, J. L. 2007 How swifts control their glide performance with morphing wings. Nature 446, 1082-1085.

Margolin, L. G., Rider, W. J. \& Grinstein, F. F. 2007 Modelling turbulent flow with implicit LES. J. Turbul. 7, 1-27.

Morton, B. R. 1984 The generation and decay of vorticity. Geophys. Astrophys. Fluid Dyn. 28, 277-308.

Müller, U. K. \& Lentink, D. 2004 Physiology - turning on a dime. Science 306, 1899-1900.

PRANDTL, L. 1927 Die Entstehung von Wirbeln in einer Flüssigkeit Kleinster Reibung. Z. Flugtech. Motorluftschiffahrt 18, 489-496.

PRANDTL, L. 1936 Entstehung von Wirbeln bei Wasserströmungen: - 1. Entstehung von Wirbeln und Künstliche Beeinflussung der Wirbelbildung. Institut für Wissenschaftlichen Film (DVD) - Historische Filmaufnahmen.

Raffel, M., Willert, C. E., Wereley, S. T. \& Kompenhans, J. 2007 Particle Image Velocimetry: A Practical Guide, 2nd edn. Springer.

RockWell, D. 1998 Vortex-body interactions. Annu. Rev. Fluid Mech. 30, 199-229.

Slaouti, A. \& Gerrard, J. H. 1981 An experimental investigation of the end effects on the wake of a circular cylinder towed through water at low Reynolds numbers. J. Fluid Mech. 112, 297-314.

Spagnolie, S. E. \& Shelley, M. J. 2009 Shape-changing bodies in fluid: hovering, ratcheting, and bursting. Phys. Fluids 21, 013103.

TANedA, S. 1977 Visual study of unsteady separated flows around bodies. Prog. Aerosp. Sci. 17, 287-348.

TAYLOR, G. I. 1953 Formation of a vortex ring by giving an impulse to a circular disk and then dissolving it away. J. Appl. Phys. 24, 104.

Wagner, H. 1925 Über die Entstehung des dynamischen Auftriebes an Tragflügeln. Z. Angew. Math. Mech. 5, 17-35.

Weymouth, G. D., Dommermuth, D. G., Hendrickson, K. \& Yue, D. K.-P. 2006 Advancements in Cartesian-grid methods for computational ship hydrodynamics. In 26th Symposium on Naval Hydrodynamics. Office of Naval Research.

Weymouth, G. D. \& Yue, D. K.-P. 2011 Boundary data immersion method for Cartesian-grid simulations of fluid-body interaction problems. J. Comput. Phys. 230, 6233-6247.

Zdravkovich, M. M. 2003 Flow Around Circular Cylinders, Volume 2: Applications. Oxford University Press. 